\title{
Compressibility of quark matter under strong magnetic field in the NJL model
}

\author{
Li Yang $\oplus^{1, *}$ and Xin-Jian Wen $\circledast^{2, \dagger}$ \\ ${ }^{1}$ Department of Physics and Electronic Engineering, Jinzhong University, Jinzhong 030619, China \\ ${ }^{2}$ Institute of Theoretical Physics, State Key Laboratory of Quantum Optics and Quantum Optics Devices, \\ Shanxi University, Taiyuan 030006, China
}

(Received 20 August 2021; revised 1 November 2021; accepted 18 November 2021; published 8 December 2021)

\begin{abstract}
The compressibility of magnetized quark matter is investigated in the SU(2) NJL model. The increases of the chemical potential and the temperature can reduce the compressibility, and lead to the much stiffer equation of state. The variation of the compressibility with the magnetic field will depend on the phase region. Due to the anisotropic structure, the compressibility is different in the directions parallel and perpendicular to the field. The discontinuity of longitudinal compressibility with the chemical potential and the temperature captures the signature of a first-order chiral phase transition and the crossover at high temperature. Moreover, the magnetic-field-and-temperature running coupling would have an important effect on the position of the phase transition. Under the lowest landau level approximation at zero temperature, the longitudinal compressibility has a direct inverse proportional relation to the magnetic field strength and the chemical potential square as $\kappa_{\mathrm{LLL}, \chi}^{\|} \propto 1 /\left(e B \mu^{2}\right)$.
\end{abstract}

DOI: 10.1103/PhysRevD.104.114010

\section{INTRODUCTION}

The properties of the quark matter are of most importance in understanding many physical aspects of nature, such as the quark gluon plasma in the big bang of the early universe, the possible structure in the core of compact objects, and the hadronic quark phase transition in experiments, where the high temperature and high densities characterize the extreme conditions. Recently, the study of properties of the quark matter is extended to a strong magnetic field [1-3], and further extended to a parallel electric and magnetic field background [4]. It is widely accepted that the strong magnetic fields could exist in the early universe, in the core of neutron stars, and in the noncentral heavy ion collision experiments, such as the Relativistic Heavy Ion Collider or the Large Hadron Collider (LHC) [5]. The magnitude of the magnetic field can reach the order of $10^{19}$ Gauss or higher in these conditions, which is much stronger than the value $10^{16}$ Gauss in some magnetars [6]. The magnetic field in the interior of stars could go up to the maximum strengths of $10^{18}-10^{20}$ Gauss [5,7]. In the experiments at the

\footnotetext{
yangl@jzxy.edu.cn

wenxj@sxu.edu.cn
}

Published by the American Physical Society under the terms of the Creative Commons Attribution 4.0 International license. Further distribution of this work must maintain attribution to the author(s) and the published article's title, journal citation, and DOI. Funded by SCOAP.
LHC/CERN energy, it is possible to produce a magnetic field of $5 \times 10^{19}$ Gauss [5,8], where all the flavors could be lying in the lowest Landau level. Quark matter under strong magnetic field has been studied extensively [9-13]. These magnetic fields are short-lived at very high energies, but play an important role in understanding the chiral magnetic effect, the possible signatures of strong $C P$ violation in experiments, and the equation of state of compact stars [1417]. Recently, the magnetic catalysis and inverse magnetic catalysis reveal the important effect of the magnetic effect on the chiral symmetry restoration. The nonzero anomalous magnetic moment of charged particle may be helpful in the realization of inverse magnetic catalysis of the chiral transition in a strong magnetic field, and it has been found to turn the chiral crossover into a first-order phase transition [18].

The equation of state (EOS) is very important to characterize the compact stars, and it has been obtained from a field theoretical approach [19]. The equation of state has demonstrated that quark stars are self-bound by the strong interaction, while neutron stars are bound by gravity $[20,21]$. In fact, the compressibility as an important aspect of the EoS can reflect the relative stiffening of the equation of state of the star. A deep understanding of the magnetic field on the equation of state can be explored by the compressibility. Up to now, few relevant researches have been directly done on quark matter under strong magnetic filed. The initial work can be returned to the two distinct concepts of the compression modulus(incompressibility) and the compressibility to reflect the stiffening of the 
equation of state of nuclear matter and QCD matter. The compression modulus stands for the ability to withstand compression. The larger value of the compression modulus, the more incompressibility. However, the compressibility is supposed as a sensitive quantity to the fluctuation of the phase transition. Its behavior is opposite to that of the compression modulus. Recently, much research has been focused on nuclear matter and the quark matter under zero magnetic field [22-26]. The compressibility of the rotating black hole has been investigated [27]. It is found that adiabatic compressibility is maximal in the extremal case and still less than the cold neutron star, but it will vanish for a nonrotating black hole. The compressibility of quark matter has been investigated in Nambu-Jona-Lasinio (NJL) model and PNJL model under zero magnetic field. It is found that the compressibility $\kappa$ is higher in NJL model than that of the PNJL model in the hadronic phase, the behavior is just the opposite in the partonic phase. So the core of the neutron star would be much softer than the crust if described by PNJL model rather than NJL model [28]. The compressibility are enhanced in the chiral symmetry broken phase and are divergent at the tricritical point in NJL model [29]. The singular behavior may be appear in the cooling process of the QGP generated in the high energy heavy-ion collisions.

The NJL model has proved to be very successful in the description of the spontaneous breaking of chiral symmetry exhibited by the true (nonperturbative) QCD vacuum, as well as many other low energy phenomena of strong interaction. In our previous work, the strong magnetic field effect has been investigated in the isospin asymmetry matter and symmetric matter [30-32]. The stronger magnetic field can enhance the spin polarization and arrange quarks in a uniform spin orientation [31]. Motivated by the work of Miransky and Shovkovy [33], it is demonstrated that the magnetic field has key effect on the coupling constant. The inverse magnetic catalysis can be realized by introducing the magnetic-field-and-temperature-dependent coupling [34-37]. A proper regularization scheme is required to deal with the divergent integral in this model. In literature, the magnetic field independent regularization (MFIR) scheme was developed in $[38,39]$ and achieved by the dimensional regularization method in Ref. [40], and extended for the presence of color superconductivity [41]. The MFIR based on the Hurwitz-Riemann zeta function was employed to study the neutral meson in hot and magnetized quark matter [42]. In this paper, our aim is to investigate the influence of the magnetic field on the compressibility of two-flavor quark matter at finite densities in the framework of SU(2)NJL model.

This work is organized as follows. In Sec. II, the thermodynamics of quark matter in a strong magnetic field is reviewed in the NJL model. In Sec. III, the numerical results and discussion are given with a detailed analysis of the compressibility of quark matter as functions of the temperature, the chemical potential and the strong magnetic fields. The last section is a short summary.

\section{THERMODYNAMICS OF MAGNETIZED QUARK MATTER IN SU(2) NJL MODEL}

The Lagrangian density of the two-flavor NJL model in a strong magnetic field is given as

$$
\mathcal{L}_{\mathrm{NJL}}=\bar{\psi}(i \not D-m) \psi+G\left[(\bar{\psi} \psi)^{2}+\left(\bar{\psi} i \gamma_{5} \vec{\tau} \psi\right)^{2}\right],
$$

where $\psi$ represents a flavor isodoublet ( $u$ and $d$ quarks), and $\vec{\tau}$ are isospin Pauli matrices. The covariant derivative $D_{\mu}=\partial_{\mu}-i Q e A_{\mu}$ represents the coupling of the quarks to the electromagnetic field, where $Q=\operatorname{diag}\left(q_{u}, q_{d}\right)=$ $\operatorname{diag}(2 / 3,-1 / 3)$ is the quark electric charge matrix in flavor space. A sum over flavor and color degrees of freedom is implicit. In the mean-field approximation [43], the dynamical quark mass is related to the condensation terms as

$$
M_{i}=m_{i}-2 G\langle\bar{\psi} \psi\rangle,
$$

where the current masses $m_{u}=m_{d}=m$ are used and the quark condensates include $u$ and $d$ quark contributions as $\langle\bar{\psi} \psi\rangle=\sum_{i=u, d} \phi_{i}$. The constituent mass depends on both condensates. Therefore, the same mass $M_{u}=M_{d}=M$ is available for $u$ and $d$ quarks. The contribution from the quark flavor $i$ is

$$
\phi_{i}=\phi_{i}^{\mathrm{vac}}+\phi_{i}^{\mathrm{mag}}+\phi_{i}^{\mathrm{med}} .
$$

The terms $\phi_{i}^{\mathrm{vac}}, \phi_{i}^{\mathrm{mag}}$, and $\phi_{i}^{\mathrm{med}}$ representing the vacuum, magnetic field, and medium contribution to the quark condensation are respectively [40,44]

$$
\begin{aligned}
& \phi_{i}^{\mathrm{vac}}=-\frac{M N_{c}}{2 \pi^{2}}\left[\Lambda \sqrt{\Lambda^{2}+M^{2}}-M^{2} \ln \left(\frac{\Lambda+\sqrt{\Lambda^{2}+M^{2}}}{M}\right)\right], \\
& \phi_{i}^{\mathrm{mag}}=-\frac{M\left|q_{i}\right| e B N_{c}}{2 \pi^{2}}\left\{\ln \left[\Gamma\left(x_{i}\right)\right]-\frac{1}{2} \ln (2 \pi)\right. \\
&\left.+x_{i}-\frac{1}{2}\left(2 x_{i}-1\right) \ln \left(x_{i}\right)\right\} \\
& \phi_{i}^{\mathrm{med}}= \sum_{k_{i}=0} a_{k_{i}} \frac{M\left|q_{i}\right| e B N_{c}}{4 \pi^{2}} \int \frac{d p}{E_{i}^{*}}\left(f_{i}^{+}+f_{i}^{-}\right)
\end{aligned}
$$

where $a_{k_{i}}=2-\delta_{k 0}$ and $k_{i}$ are respectively the degeneracy label and the Landau quantum number. The dimensionless quantity $x_{i}$ is defined as $x_{i}=M^{2} /\left(2\left|q_{i}\right| e B\right)$. The fermion distribution function is 


$$
f_{i}^{ \pm}=\frac{1}{1+\exp \left[\left(E_{i}^{*} \mp \mu_{i}\right) / T\right]} .
$$

The effective quantity $E_{i}^{*}=\sqrt{p^{2}+s_{i}^{2}}$ sensitively depends on the magnetic field through $s_{i}=\sqrt{M^{2}+2 k_{i}\left|q_{i}\right| e B}$. The chemical potential $\mu_{u}=\mu_{d}=\mu$ is achieved for the isospin symmetry. The quark condensation is greatly strengthened by the factor $\left|q_{i} e B\right|$ together with the dimension reduction $D-2[33,45]$. The total thermodynamic potential density in the mean field approximation reads

$$
\Omega=\frac{(M-m)^{2}}{4 G}+\sum_{i=u, d} \Omega_{i},
$$

where the first term is the interaction term. In the second term, the quantity is defined as $\Omega_{i}=\Omega_{i}^{\mathrm{vac}}+\Omega_{i}^{\mathrm{mag}}+\Omega_{i}^{\mathrm{med}}$. The vacuum contribution to the thermodynamic potential is

$$
\Omega_{i}^{\mathrm{vac}}=\frac{N_{c}}{8 \pi^{2}}\left[M^{4} \ln \left(\frac{\Lambda+\epsilon_{\Lambda}}{M}\right)-\epsilon_{\Lambda} \Lambda\left(\Lambda^{2}+\epsilon_{\Lambda}^{2}\right)\right],
$$

where the quantity $\epsilon_{\Lambda}$ is defined as $\epsilon_{\Lambda}=\sqrt{\Lambda^{2}+M^{2}}$. The ultraviolet divergence in the vacuum part $\Omega_{i}^{\mathrm{vac}}$ of the thermodynamic potential is removed by the momentum cutoff. The magnetic field and medium contributions are respectively

$$
\Omega_{i}^{\mathrm{mag}}=-\frac{N_{c}\left(\left|q_{i}\right| e B\right)^{2}}{2 \pi^{2}}\left[\zeta^{\prime}\left(-1, x_{i}\right)-\frac{1}{2}\left(x_{i}^{2}-x_{i}\right) \ln \left(x_{i}\right)+\frac{x_{i}^{2}}{4}\right],
$$

$$
\begin{aligned}
\Omega_{i}^{\mathrm{med}}= & -T \sum_{k=0} a_{k_{i}} \frac{N_{c}\left|q_{i}\right| e B}{4 \pi^{2}} \int d p\left\{\ln \left[1+\exp \left(-\frac{E_{i}^{*}-\mu}{T}\right)\right]\right. \\
& \left.+\ln \left[1+\exp \left(-\frac{E_{i}^{*}+\mu}{T}\right)\right]\right\}
\end{aligned}
$$

where $\zeta(x, a)=\sum_{n=0}^{\infty} \frac{1}{(n+a)^{x}}$ is the Hurwitz zeta function. From the thermodynamic potential (8), one can easily obtain the quark density as

$$
n_{i}(\mu, B)=\sum_{k=0} a_{k_{i}} \frac{N_{c}\left|q_{i}\right| e B}{4 \pi^{2}} \int d p\left(f_{i}^{+}-f_{i}^{-}\right) .
$$

It is widely known that the strong magnetic fields can have significant impact on the thermodynamic properties of quark matter. Importantly, the breaking of the rotational symmetry produces the anisotropic structure, with the parallel pressure $P^{\|}$and the perpendicular pressure $P^{\perp}$ in directions along and transverse to the magnetic field respectively [9,46-49],

$$
\begin{gathered}
P^{\|}=-\Omega-\frac{B^{2}}{2}, \\
P^{\perp}=-\Omega-\mathcal{M} B+\frac{B^{2}}{2},
\end{gathered}
$$

where the magnetization is $\mathcal{M}=-\left(\frac{\partial \Omega}{\partial B}\right)_{\mu}$. Consequently, the pressure anisotropy will affect the determination of the compressibility. In the thermodynamics, the isothermal compressibility is defined as

$$
\kappa=-\frac{1}{V}\left(\frac{\partial V}{\partial P}\right)_{T}
$$

which measures how much the volume of the system decreases with increase of pressure. The minus is generally used for a positive quantity. Its smaller value indicates the matter is stiffer. The compressibility of quark matter can be derived from the potential to reflect the fluctuation of the order parameter sensitive to the phase transition. The longitudinal compressibility can be written in a tractable form as [29]

$$
\kappa^{\|}=-\frac{1}{V}\left(\frac{\partial V}{\partial n_{q}}\right)\left(\frac{\partial n_{q}}{\partial P^{\|}}\right)_{T}=\frac{1}{n_{q}^{2}}\left(\frac{\partial n_{q}}{\partial \mu_{q}}\right)_{T},
$$

where $n_{q}$ is the quark number density and $\mu_{q}=\frac{\mu_{u}+\mu_{d}}{2}$ is the quark chemical potential. Our compressibility reflects the thermodynamical behavior opposite to the so-called compressibility modulus $K=k_{F}^{2} d^{2}\left(\varepsilon / n_{q}\right) / d k_{F}^{2}$, which stands for the ability to withstand compression. The transverse compressibility in the direction perpendicular to the field is

$$
\kappa^{\perp}=\frac{1}{n_{q}}\left(\frac{\partial n_{q}}{\partial P^{\perp}}\right)_{T}
$$

The compressibility could manifest the anisotropic structure due to the breaking of the rotation symmetry. The transverse compressibility would depend on the magnetization $\mathcal{M}$ in the strong magnetic field.

\section{NUMERICAL RESULTS AND DISCUSSION}

In the present calculation, the following parameters are adopted: $m_{u}=m_{d}=5.6 \mathrm{MeV}, \quad \Lambda=587.9 \mathrm{MeV}$, and $G=2.44 / \Lambda^{2}$. The quark dynamical mass should be solved by the gap equation (2). In Fig. 1, the longitudinal compressibility are shown as the function of the chemical potential for a fixed temperature $T=150 \mathrm{MeV}$. The solid, dashed, and dotted lines represent the magnetic fields $B=0,5 \times 10^{18}, 5 \times 10^{19}$ Gauss, respectively. It can be clearly seen that the magnetic field makes the compressibility become little, and consequently results in a stiff equation of state. Especially at smaller chemical potential, the compressibility would sensitively depend on the 


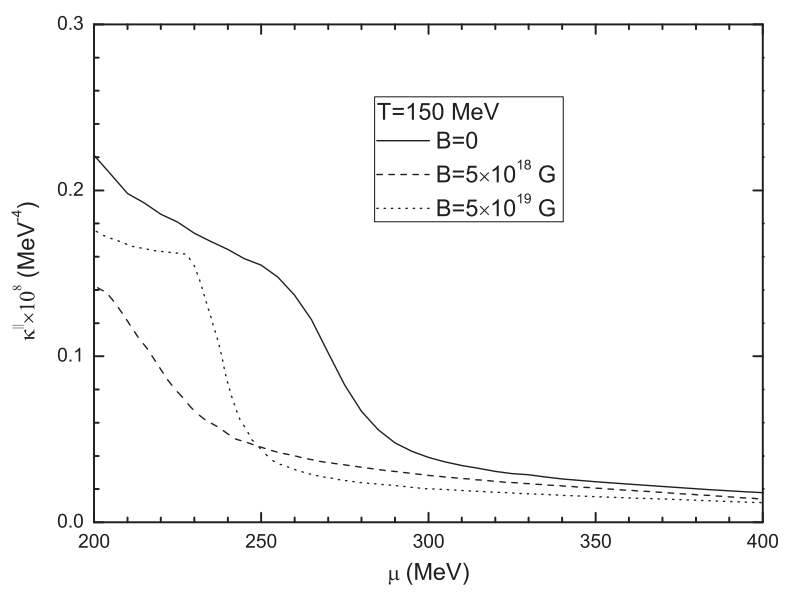

FIG. 1. The longitudinal compressibility versus the chemical potential at different magnetic fields and a fixed temperature.

magnetic field strength. At larger chemical potential of the chiral restored phase, the magnetic effect becomes weak. This feature can be understood by the fact that in chiral restored phase, the quark number density is much higher and the stiffness of the equation of state cannot change evidently any more. To some extent, it can be predicted that the magnetic field would lead to much stiffer equation of state in neutron stars compared to zero magnetic field condition.

In Fig. 2, we show the dynamical quark mass on the left panel and the compressibility on the right panel as the functions of the chemical potential for a fixed magnetic field $B=5 \times 10^{19}$ Gauss. In order to investigate the thermal effect on the compressibility, the different temperatures $T=100,150,200 \mathrm{MeV}$ are marked by the solid, dashed, and dotted lines, respectively. It is clear that the higher the temperature the less the compressibility at a fixed magnetic field. So it is expected that the increase of

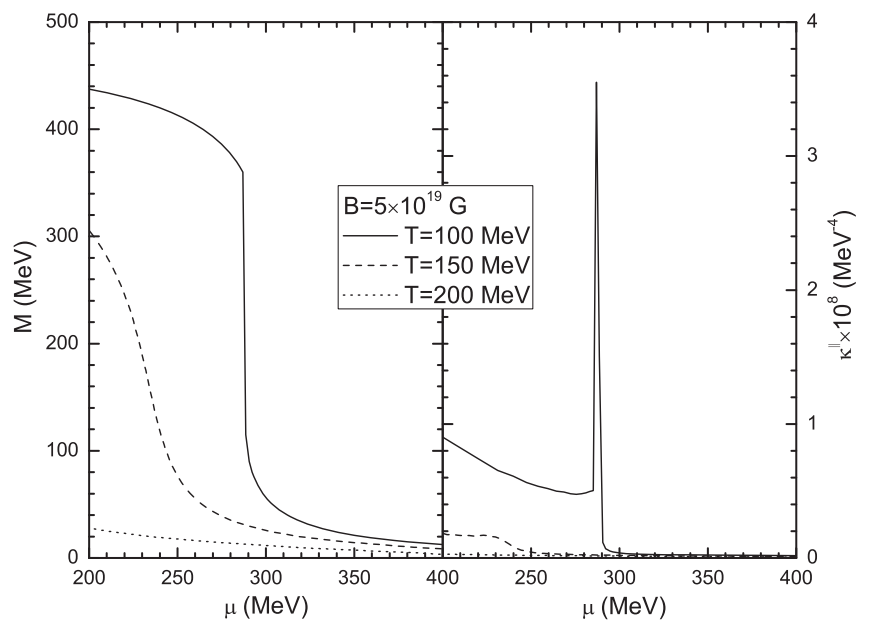

FIG. 2. The longitudinal compressibility as a function of the chemical potential for the fixed magnetic fields and three different temperatures.

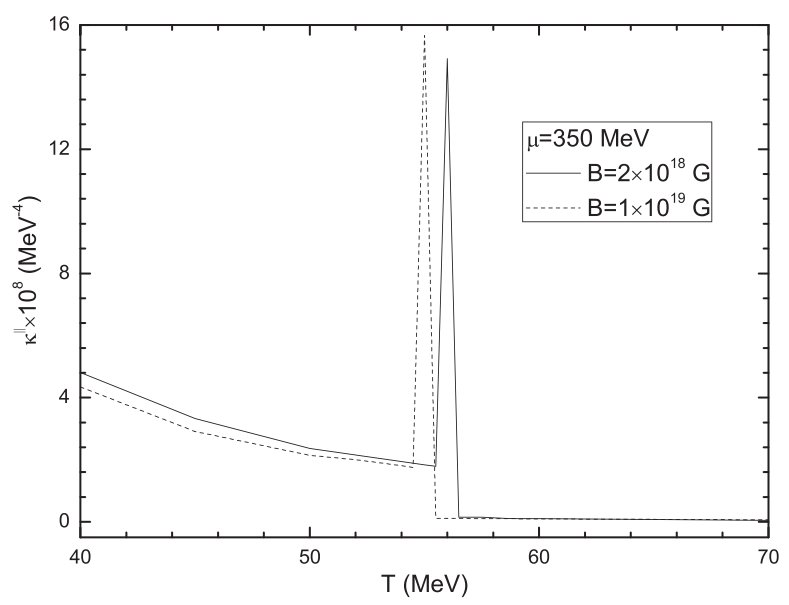

FIG. 3. The longitudinal compressibility as a monotonous decreasing function of the temperature at the chemical potential $\mu=350 \mathrm{MeV}$ and the magnetic fields $B=2 \times 10^{18}$ Gauss (solid line) and $B=1 \times 10^{19}$ Gauss (dashed line).

the temperature and/or the chemical potential can make the quark matter become much stiffer. At low temperature marked by the solid line, the compressibility changes obviously as the chemical potential. The decreasing compressibility shows a sharp cusp indicating the critical chemical potential of the first order transition, which is consistent with the sharp drop of quark mass on the left panel. However at higher temperatures, the compressibility would remain constant and hardly change with the increasing chemical potential anymore.

To show the detailed behavior of the compressibility, we show the compressibility as the function of the temperature at a fixed chemical potential $\mu=350 \mathrm{MeV}$ and the two magnetic fields $2 \times 10^{18}$ and $1 \times 10^{19}$ Gauss in Fig. 3. The compressibility evidently decreases as the temperature increases and the descent behavior would slow down in the high density region. The peak of the compressibility indicates the first-order transition of the chiral restoration at high densities. By comparison of the two peaks, it is indicated that for the stronger magnetic fields, the chiral phase transition happens at lower temperature. It is concluded that the stiffness of the equation of state can be dominantly enhanced by the increase of the temperature at fixed chemical potentials.

In Fig. 4, the longitudinal compressibility versus the magnetic field is shown at a high temperature $T=150 \mathrm{MeV}$ and the low chemical potential $\mu=260 \mathrm{MeV}$, the value of the compressibility raises from a small value to a maximum and then descends rapidly as the increasing magnetic field. The peak position of the curve implies the chiral crossover at $\left(T_{c}=150 \mathrm{MeV}, \mu_{c}=260 \mathrm{MeV}\right)$ with the corresponding critical magnetic field $B_{c}=8.1 \times$ $10^{19}$ Gauss. From the figure we can see that a slight perturbation of the compressibility appears due to the small fluctuation of the order parameter at a fixed chemical 


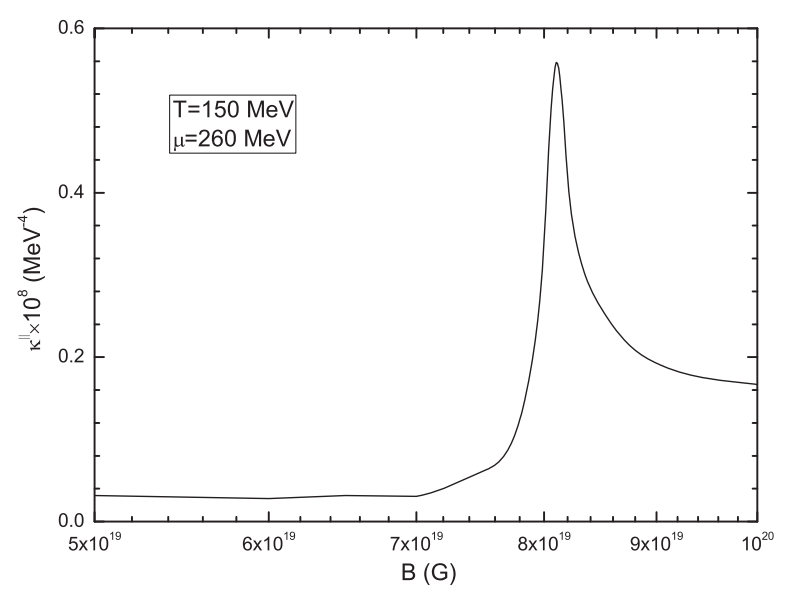

FIG. 4. Variation of longitudinal compressibility with the magnetic field at the temperatures $T=150 \mathrm{MeV}$ and the chemical potential $\mu=260 \mathrm{MeV}$.

potential. The peak position could tell the precise value of the magnetic field, at which the chiral crossover occurs at the fixed temperature and chemical potential.

From the definition in Eq. (16), it is clear that the compressibility $\kappa$ involves the quark number susceptibility (QNS), namely, the derivation of the quark number density with the chemical potential $\left(\partial n_{q} / \partial \mu_{q}\right)$. In literature, the behavior of the QNS was suggested to indicate the phase transition [50]. In Fig. 5, the quark number density and the QNS with the chemical potential are shown. For the convenience of labeling the axis, the factors $10^{6}$ and $10^{5}$ are multiplied in the production respectively. The solid, dashed, dotted, and dash-dotted lines stand for the magnetic fields $7 \times 10^{19}, 7.5 \times 10^{19}$, $8.1 \times 10^{19}$, and $8.5 \times 10^{19}$ Gauss. From the numerical result, it is expected that the quark number density

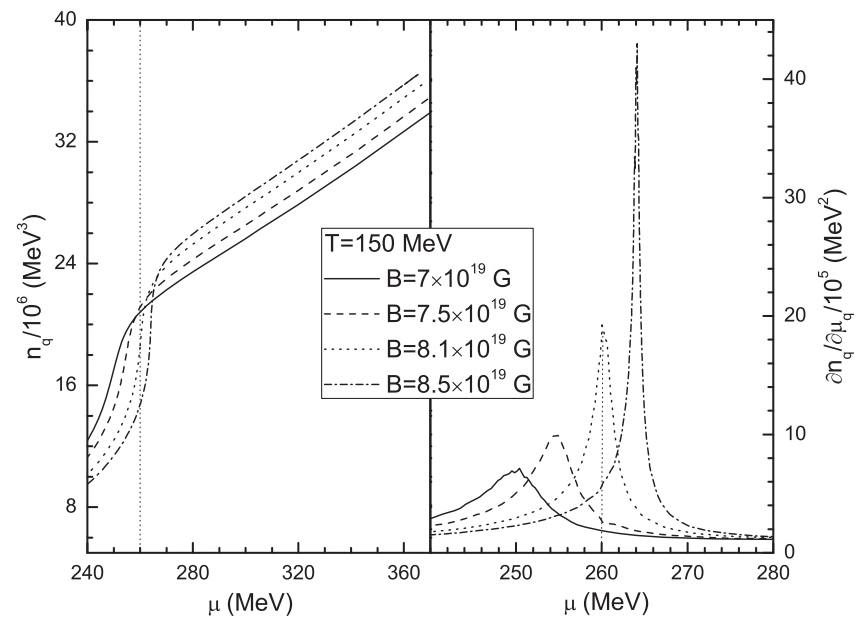

FIG. 5. The quark number density $n_{q}$ and the derivatives $\partial n_{q} / \partial \mu_{q}$ versus the chemical potential for a fixed temperatures $T=150 \mathrm{MeV}$ and four different magnetic fields $B=7 \times 10^{19}$, $7.5 \times 10^{19}, 8.1 \times 10^{19}, 8.5 \times 10^{19}$ Gauss. increases as the chemical potential increases. In the ascending lines on the left panel, there is a inflection point, which can be identified by the peak of the QNS on the right panel. The peak position of the QNS appears at $\left(\mu_{c}=260 \mathrm{MeV}, B_{c}=8.1 \times 10^{19}\right.$ Gauss $)$, which exactly implies the occurrence of the chiral crossover. It is also responsible for the peak in Fig. 4. The increasing magnetic field moves the peak position toward the high density, which reflects the magnetic catalysis for the pseudocritical chemical potential.

In principle, the interaction coupling constant should be solved by the renormalization group equation, or be phenomenologically expressed in an effective potential dependent on environmental variables. In the presence of a strong magnetic field, it is well known that the interaction constant shows an obvious decreasing behavior in addition to the enlargement of the gluon mass, which accounts for the inverse magnetic catalysis. To obtain a reasonable description of the temperature dependence of the interaction, we employ the running coupling constant $G(B, T)$ from the lattice simulations [34]. In Figs. 6 and 7, the quark density and the derivative are plotted versus the chemical potentials. At low temperature $T=50 \mathrm{MeV}$ in Fig. 6, the peaks of the QNS indicate the first-order phase transition at the magnetic fields $B=5 \times 10^{19}, 1 \times 10^{20}$ Gauss and the crossover at the fields $B=2 \times 10^{18}, 2 \times 10^{19}$ Gauss. The increasing magnetic fields move the pseudocritical chemical potential to the lower value for the chiral crossover, which indicates the inverse magnetic catalysis. On the contrary, the magnetic catalysis on the first-order transition is found that the critical chemical potential is pushed to high value by the magnetic field. In Fig. 7, at the fixed magnetic field $B=2.0 \times 10^{19}$ Gauss, the thermal effect is investigated on quark number density and the QNS.

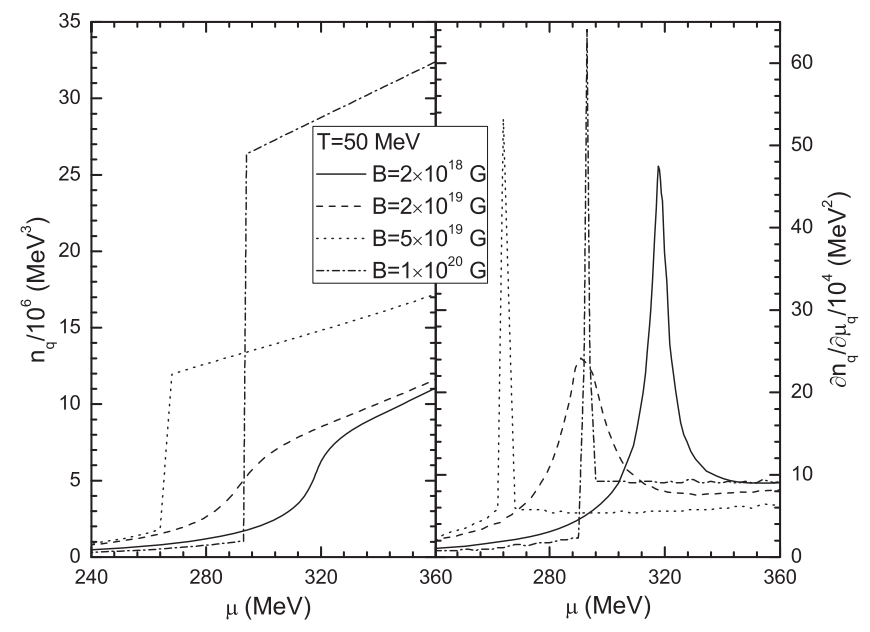

FIG. 6. The quark number density $n_{q}$ and the derivatives $\partial n_{q} / \partial \mu_{q}$ versus the chemical potential for a fixed temperatures $T=50 \mathrm{MeV}$ and four different magnetic fields $B=2 \times 10^{18}$, $2 \times 10^{19}, 5 \times 10^{19}, 1 \times 10^{20}$ Gauss. 


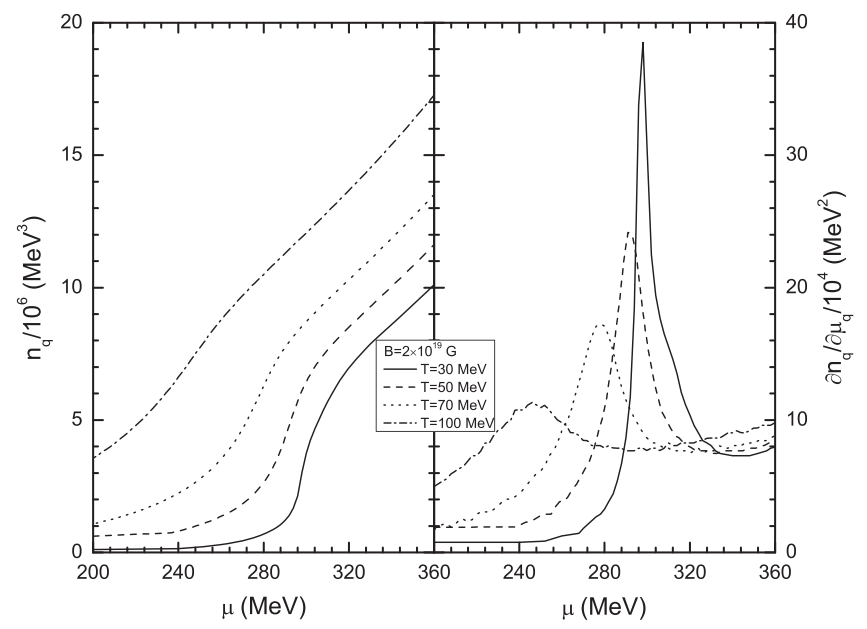

FIG. 7. The quark number density $n_{q}$ and the derivatives $\partial n_{q} / \partial \mu_{q}$ versus the chemical potential for a fixed magnetic field $B=2 \times 10^{19}$ Gauss and the four temperature $T=30,50,70$, $100 \mathrm{MeV}$.

The different temperatures $T=30,50,70$, and $100 \mathrm{MeV}$ are marked by the solid, the dashed, the dotted, and the dash-dotted lines, respectively. The peaks of the QNS indicate the pseudocritical chemical potential of the crossover is expected to be reduced by the increase of the temperature.

It is generally accepted that the strong magnetic field higher than the order of $10^{19}$ Gauss would make the quarks occupy the lowest landau level (LLL). Then the quark number density at $T=0$ reads,

$$
n_{i}^{\mathrm{LLL}}\left(\mu_{i}, B\right)=\frac{N_{c}\left|q_{i}\right| e B}{4 \pi^{2}} \sqrt{\mu_{i}^{2}-M_{i}^{2}} .
$$

The corresponding derivative of the number density with respect to the chemical potential becomes

$$
\frac{\partial n_{i}^{\mathrm{LL}}}{\partial \mu_{i}}=\frac{N_{c}\left|q_{i}\right| e B}{4 \pi^{2}} \frac{\mu_{i}}{\sqrt{\mu_{i}^{2}-M_{i}^{2}}}
$$

Therefore, in extreme strong magnetic fields, the isospinsymmetric quarks in the lowest Landau level contribute to the longitudinal compressibility in a analytical form,

$$
\kappa_{\mathrm{LLL}}^{\|}=\frac{2 \pi^{2}}{N_{c}\left(\left|q_{u}\right|+\left|q_{d}\right|\right) e B} \frac{\mu}{\left(\mu^{2}-M^{2}\right)^{3 / 2}} .
$$

In the chiral limit, the dynamical quark mass vanishes and the compressibility becomes more simply dependent on the chemical potential,

$$
\kappa_{\mathrm{LLL}, \chi}^{\|}=\frac{2 \pi^{2}}{N_{c}\left(\left|q_{u}\right|+\left|q_{d}\right|\right) e B \mu^{2}} .
$$

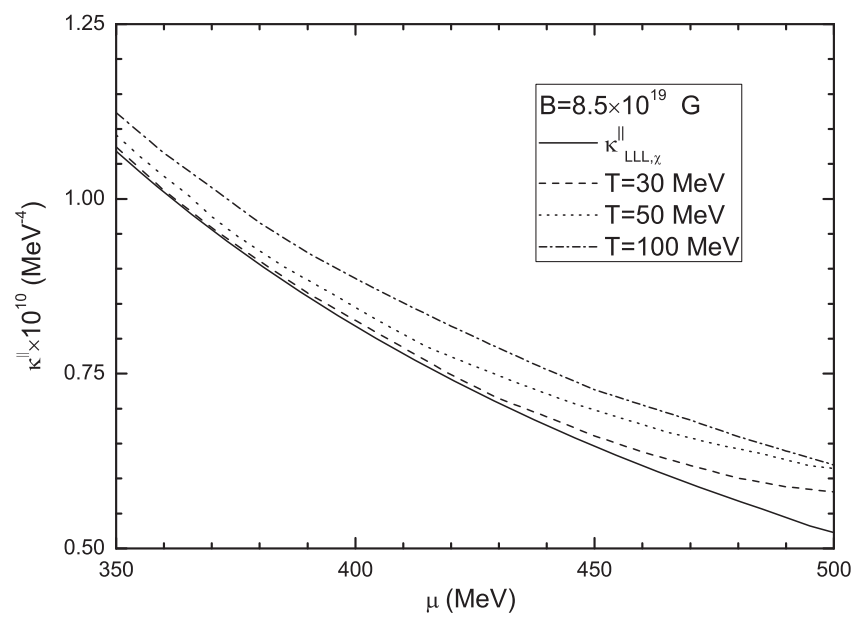

FIG. 8. The longitudinal compressibility $\kappa^{\|}$versus the chemical potential for the magnetic field $B=8.5 \times 10^{19}$ Gauss and three different temperatures $T=30,50,100 \mathrm{MeV}$. The solid line indicates the compressibility $\kappa_{\mathrm{LLL}, \chi}^{\|}$at zero temperature in the chiral limit and the LLL approximation.

which means that the condition of both the stronger magnetic field and larger chemical potential would directly result in the smaller compressibility for the bulk matter.

In Fig. 8, the longitudinal compressibility $\kappa^{\|}$is plotted as a function of the chemical potential at a strong magnetic field close to the order $10^{20}$ Gauss, which satisfies the lowest Landau level approximation. The degeneracy factor proportional to the field strength $|e B|$ accounts for the larger number of quarks. The longitudinal compressibility is a decreasing function of the chemical potential at high densities. Moreover, it is clear that the compressibility will gradually approach to the $\kappa_{\mathrm{LLL},}^{\|}$ marked by the solid line as the temperature goes down to zero. In the LLL approximation, the compressibility of the chiral restoration phase at $T=0$ is inversely proportional to the chemical potential square. Therefore, it is concluded that the increases of the chemical potential will reduce the compressibility and lead to the much stiffer equation of state.

The transverse compressibility as a function of the quark chemical potential is shown in Fig. 9 at the magnetic field $B=8.5 \times 10^{19}$ Gauss. The descending transverse compressibility versus the chemical potential behaves similarly to the longitudinal compressibility. However, the anisotropic structure induced by the external magnetic field would have important effect on the compressibility. In Fig. 10, The longitudinal compressibility $\kappa^{\|}$and the transverse term $\kappa^{\perp}$ are plotted at the magnetic fiend $B=$ $1.0 \times 10^{20}$ Gauss and the temperature $T=50 \mathrm{MeV}$. The $\kappa^{\perp}$ marked by the solid line is much larger than the transverse term marked by the dashed line. But the difference between them would gradually become unobvious at larger chemical potentials. 


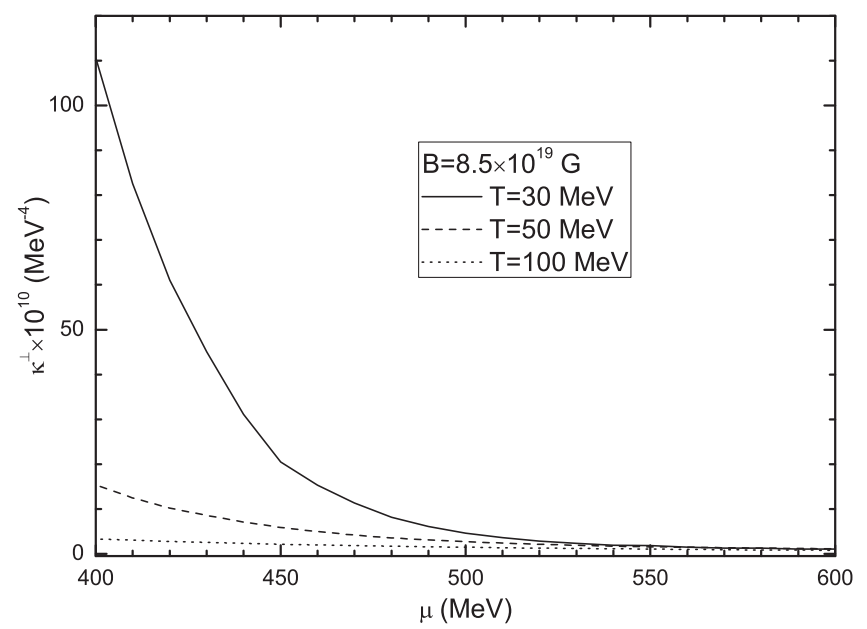

FIG. 9. The transverse compressibility $\kappa^{\perp}$ perpendicular to the magnetic field at $B=8.5 \times 10^{19}$ Gauss and three different temperatures $T=30,50,100 \mathrm{MeV}$.

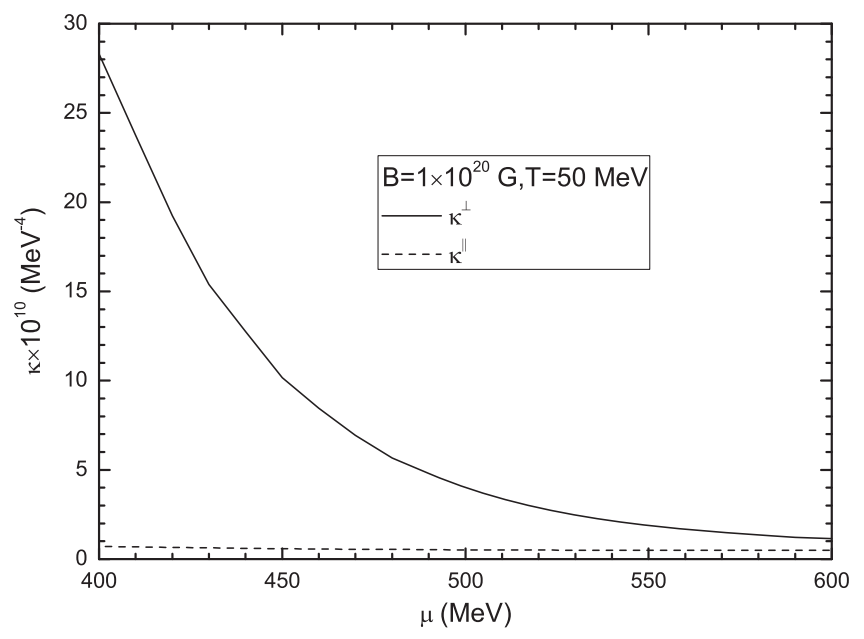

FIG. 10. The anisotropic compressibility $\kappa$ versus the chemical potential for the magnetic field $B=1.0 \times 10^{20}$ Gauss and the temperature $T=50 \mathrm{MeV}$.

\section{SUMMARY}

In this paper we have studied the compressibility of 2-flavor quark matter in a strong magnetic field within the SU(2) NJL model. The compressibility is defined as a variable to show how the volume changes with the increases of the pressure. The value can reflect the stiffness of the equation of state of quark matter. A deep investigation on the compressibility is helpful to further understanding the phase transition at the finite temperature, high densities, and the strong magnetic fields. It is found that the magnetic field can make the quark matter become stiffer by reducing the compressibility. However, the variation of the compressibility with the magnetic field depends on the phase that we are interested in. The variation of the longitudinal compressibility with the chemical potential shows a sharp cusp indicating a firstorder chiral phase transition. For the high temperature and the low chemical potential, the peak of the longitudinal compressibility is responsible for the critical magnetic field of the chiral crossover.

Specially, the inflection points of the quark number density and the corresponding peak position of the quark number susceptibility $\partial n_{q} / \partial \mu_{q}$ indicate the critical chemical potential. By employing the magnetic-fieldand-temperature-dependent running coupling constant, first it is found that the increasing magnetic field would change the chiral crossover to the first order transition at low temperature. Second, it is expected that the increase of the temperature will lead to the decreasing of the pseudocritical chemical potential for the crossover. The breaking of the rotation leads to the anisotropic compressibility. It is found that the transverse compressibility $\kappa^{\perp}$ is much larger than the longitudinal term $\kappa^{\|}$. The difference between the $\kappa^{\perp}$ and $\kappa^{\|}$becomes unobvious at larger chemical potential. In the very strong magnetic fields, all quarks are lying in the lowest landau level. At zero temperature, it has been found that the longitudinal compressibility in the chiral restoration phase will be inversely proportional to the magnetic field and chemical potential square, namely, $\kappa_{\mathrm{LLL}, \chi}^{\|} \propto 1 /\left(e B \mu^{2}\right)$. The analytical relation demonstrates that the increase of the chemical potential leads to the smaller compressibility and the stiffer equation of state. We hope that the future investigation of the compressibility of quark matter can provide a complementary sight to the QCD phase at finite densities and temperatures.

\section{ACKNOWLEDGMENTS}

The authors would like to thank support from the Scientific and Technological Innovation Programs of Higher Education Institutions in Shanxi under the Grant No. 2020L0601 and the National Natural Science Foundation of China (No. 11475110, No. 11875181, and No. 11705163). 
[1] V. A. Miransky and I. A. Shovkovy, Phys. Rep. 576, 1 (2015); J. O. Andersen, W. R. Naylor, and A. Tranberg, Rev. Mod. Phys. 88, 025001 (2016).

[2] V. P. Gusynin, V. A. Miransky, and I. A. Shovkovy, Phys. Rev. Lett. 73, 3499 (1994); E. J. Ferrer and V. de la Incera, Phys. Lett. B 481, 287 (2000); N. Mueller and J. M. Pawlowski, Phys. Rev. D 91, 116010 (2015).

[3] R. G. Felipe and A. P. Martínez, J. Phys. G 36, 075202 (2009); R. González Felipe, D. Manreza Paret, and A. Pérez Martínez, Eur. Phys. J. A 47, 1 (2011).

[4] M. Ruggieri and G.-X. Peng, Phys. Rev. D 93, 094021 (2016).

[5] D. E. Kharzeev, L. D. McLerran, and H. J. Warringa, Nucl. Phys. A803, 227 (2008).

[6] C. Thompson and R. C. Duncan, Astrophys. J. 392, L9 (1992).

[7] L. Dong and S. L. Shapiro, Astrophys. J. 383, 745 (1991).

[8] V. Voronyuk, V. D. Toneev, W. Cassing, E. L. Bratkovskaya, V. P. Konchakovski, and S. A. Voloshin, Phys. Rev. C 83, 054911 (2011).

[9] D. P. Menezes, M. B. Pinto, and C. Providência, Phys. Rev. C 91, 065205 (2015).

[10] Y. Wang and X. J. Wen, J. Phys. G 47, 105201 (2020).

[11] S. I. Nam and C.-W. Kao, Phys. Rev. D 83, 096009 (2011).

[12] S. Rechenberger, Phys. Rev. D 95, 054013 (2017).

[13] E. S. Fraga and A. J. Mizher, Phys. Rev. D 78, 025016 (2008).

[14] D. Kharzeev, R. D. Pisarski, and M. H. G. Tytgat, Phys. Rev. Lett. 81, 512 (1998); D. Kharzeev and R. D. Pisarski, Phys. Rev. D 61, 111901(R) (2000); K. Buckley, T. Fugleberg, and A. Zhitnitsky, Phys. Rev. Lett. 84, 4814 (2000); S. A. Voloshin, Phys. Rev. C 62, 044901 (2000); D. Kharzeev, Phys. Lett. B 633, 260 (2006); D. Kharzeev and A. Zhitnitsky, Nucl. Phys. A797, 67 (2007).

[15] D. E. Kharzeev, Nucl. Phys. A830, 543c (2009); Ann. Phys. (Berlin) 325, 205 (2010); K. Fukushima, D. E. Kharzeev, and H. J. Warringa, Nucl. Phys. A836, 311 (2010); Phys. Rev. Lett. 104, 212001 (2010).

[16] K. Fukushima, D. E. Kharzeev, and H. J. Warringa, Phys. Rev. D 78, 074033 (2008); D. E. Kharzeev and H. J. Warringa, Phys. Rev. D 80, 034028 (2009).

[17] V. Skokov, A. Illarionov, and V. Toneev, Int. J. Mod. Phys. A 24, 5925 (2009).

[18] X. J. Wen, R. He, and J. B. Liu, Phys. Rev. D 103, 094020 (2021).

[19] A. Broderick, M. Prakash, and J. M. Lattimer, Astrophys. J. 537, 351 (2000).

[20] D. P. Menezes and D. B. Melrose, Pub. Astron. Soc. Aust. 22, 292 (2005).

[21] D. Bandyopadhyay, S. Chakrabarty, and S. Pal, Phys. Rev. Lett. 79, 2176 (1997).

[22] J. P. Blaizot, D. Gogny, and B. Grammaticos, Nucl. Phys. A265, 315 (1976).

[23] J. P. Blaizot, Phys. Rep. 64, 171 (1980).
[24] D. T. Khoa and W. Oertzen, Phys. Lett. B 342, 6 (1995).

[25] H. Kouno, N. Kakuta, N. Noda, K. Koide, T. Misumori, A. Hasegawa, and M. Nakano, Phys. Rev. C 51, 1754 (1995).

[26] V. A. Dexheimer, C. A.Z. Vasconcellos, and B. E. J. Bodmann, Int. J. Mod. Phys. D 16, 269 (2007).

[27] B. P. Dolan, Phys. Rev. D 84, 127503 (2011).

[28] A. Bhattacharyya, S. K. Ghosh, S. Majumder, and R. Ray, Phys. Rev. D 86, 096006 (2012).

[29] M. Iwasaki, Phys. Rev. D 70, 114031 (2004).

[30] L. Yang and X. J. Wen, Int. J. Mod. Phys. A 33, 1850123 (2018).

[31] L. Yang and X. J. Wen, Commun. Theor. Phys. 67, 535 (2017).

[32] L. Yang and X. J. Wen, Phys. Rev. D 96, 056023 (2017).

[33] V. A. Miransky and I. A. Shovkovy, Phys. Rev. D 66, 045006 (2002).

[34] R. L. S. Farias, K. P. Gomes, G. I. Krein, and M. B. Pinto, Phys. Rev. C 90, 025203 (2014).

[35] M. Ferreira, P. Costa, O. Lourenço, T. Frederico, and C. Providência, Phys. Rev. D 89, 116011 (2014).

[36] J. O. Andersen, W. R. Naylor, and A. Tranberg, J. High Energy Phys. 02 (2015) 042.

[37] R. L. S. Farias, V. S. Timoteo, S. S. Avancini, M. B. Pinto, and G. Krein, Eur. Phys. J. A 53, 101 (2017).

[38] D. Ebert, K. G. Klimenko, M. A. Vdovichenko, and A. S. Vshivtsev, Phys. Rev. D 61, 025005 (1999); M. A. Vdovichenko, A. S. Vshivtsev, and K. G. Klimenko, Phys. At. Nucl. 63, 470 (2000).

[39] I. E. Frolov, V. C. Zhukovsky, and K. G. Klimenko, Phys. Rev. D 82, 076002 (2010).

[40] D. P. Menezes, M. B. Pinto, S. S. Avancini, A. P. Martínez, and C. Providência, Phys. Rev. C 79, 035807 (2009); M. Ferreira, P. Costa, D. P. Menezes, C. Providência, and N. N. Scoccola, Phys. Rev. D 89, 016002 (2014).

[41] P. G. Allen, A. G. Grunfeld, and N. N. Scoccola, Phys. Rev. D 92, 074041 (2015).

[42] S. S. Avancini, R. L. S. Farias, and W. R. Tavares, Phys. Rev. D 99, 056009 (2019).

[43] C. Ratti, Europhys. Lett. 61, 314 (2003); M. Buballa and M. Oertel, Phys. Lett. B 457, 261 (1999).

[44] S. S. Avancini, D. P. Menezes, and C. Providência, Phys. Rev. C 83, 065805 (2011).

[45] T. Kojo and N. Su, Nucl. Phys. A931, 763 (2014).

[46] M. Strickland, V. Dexheimer, and D. P. Menezes, Phys. Rev. D 86, 125032 (2012).

[47] E. J. Ferrer and A. Hackebill, Phys. Rev. C 99, 065803 (2019).

[48] B. Karmakar, R. Ghosh, A. Bandyopadhyay, N. Haque, and M. G. Mustafa, Phys. Rev. D 99, 094002 (2019).

[49] S. S. Avancini, V. Dexheimer, R. L. S. Farias, and V. S. Timóteo, Phys. Rev. C 97, 035207 (2018).

[50] M. He, J. F. Li, W. M. Sun, and H. S. Zong, Phys. Rev. D 79, 036001 (2009). 